\title{
Compare and Measure Selfishness Detection (CMSD) mechanism: promptness and accuracy
}

\begin{abstract}
Node in wireless multihop networks requires cooperation from peer neighbours to relay packets towards intended destination in ensuring optimal rate of successful data transmission. However, willingness of node to cooperate cannot be guaranteed due to the need to conserve resources that compelling selfish behaviour. Thus, node forwarding behaviour needs to be evaluated to detect selfishness and enforce punishment. Ironically, accurate evaluation of node behaviour is commonly affected by false judgment due to stochastic perceptions of an observer node especially when it is done using single set of actions (SSA) approach. In this paper, we evaluate the effectiveness of an enhanced mechanism of SSA named Compare and Measure Selfishness Detection (CMSD) based on its promptness and accuracy elements in detecting selfish node. Catering several network scenarios, our analysis shows that the promptness element introduced in CMSD is able to provide more complete behaviour information at reasonable delay trade-off in comparison to SSA, which requires extra observation sessions and induce the need of getting global opinions to collect node behavioural history. In addition, by using CMSD, node behaviour can be identified more accurately and false judgment can be detected earlier as demonstrated using our proposed matrix of judgment. We also propose a general framework of an extended version of CMSD named CMSD with Evidence (CMSDE) and provide brief explanation.
\end{abstract}

Keyword: Accuracy; Cooperation; Evidence; Flexible judgment; Promptness; Selfishness; Wireless multihop networks 